\title{
103 CHALLENGES OF PREVENTING NON COMMUNICABLE DISEASES RISK FACTORS IN URBAN CONTEXT IN TUNISIA
}

Hassen Belgacem Ghannem. Department of Epidemiology, University Hospital Farhat Hached, Sousse, Sousse, Tunisia

\subsection{6/bmjopen-2015-forum2015abstracts. 103}

Background Like most North African countries, Tunisia, is going through an epidemiologic transition with rising of life expectancy and adoption of new lifestyles that lead to Non communicable diseases (NCDs). We know that NCDs arises from three main risk factors-tobacco use, poor diet, and physical inactivity. The need for comprehensive intervention to reduce their risk factors is urgent.

Objectives to evaluate the feasibility and effectiveness of a 3 years community based intervention to prevent NCDs risk factors.

Methods The study design was a quasi experimental design, intervention with pre post assessment and control group. The intervention group including different settings (schools, workplaces) was located in the delegation of Sousse Jawhara and Sousse Erriadh. The control group with the same settings was located in the delegation of Msaken from the region of Sousse.

Sample size calculation in the different settings was based on a significance level of $\alpha=0.05 \%$, power of test $\beta=20 \%$, two sided test of hypothesis and 6\% change in risk factors levels (smoking, unhealthy diet and physical inactivity) giving a total 
sample needed of 4000 schoolchildren in school settings, 2000 workers in workplaces and 2000 adults from households. We used biometric measures (weight, height, blood pressure) and pretested questionnaire to evaluate knowledge of, attitudes towards and beliefs on the three risk factors for NCDs: unhealthy diet, physical inactivity and tobacco use.

Result In schools, fruits and vegetables consumption improved in intervention group but not in control group. In fact, the proportion of schoolchildren consuming vegetables daily increased from $28.4 \%$ to $32.4 \%(\mathrm{p}=0.008)$ and consumption of fruits daily from $55.9 \%$ to $59.3 \%(p=0.03)$. We noticed a decrease of tobacco use in intervention group but not in control group. Physical activity didn't increase in the two groups. In workplaces, tobacco use decreased but not significantly both in intervention and control group. Consumption of five fruits and vegetables daily and practice of recommended level of physical activity increased significantly in the two groups. The prevalence of hypertension decreased significantly from $16 \%$ to $12.3 \%$ $(\mathrm{p}=0.02)$ in intervention group but increased in control group from $14.2 \%$ to $22.5 \%$. In the community, there was an increase in fruits and vegetables consumption and in physical activity in both the intervention and control group. There was a decrease in tobacco consumption in the intervention group $(52.9 \%$ to $45.6 \%)$ compared to an increase in the control group $(46.9 \%$ to $50.4 \%$ ) among men.

Conclusion Targeting behavioral changes only through educative approach without environmental actions and community mobilization that facilitate healthy choices for individual is not enough.More structural changes through multisectoral intervention are needed to improve the adoption of healthy lifestyle habits and reduce the burden on individuals. 